Network Working Group

Request for Comments: 1333
W. Simpson

Daydreamer

May 1992

PPP Link Quality Monitoring

Status of this Memo

This RFC specifies an IAB standards track protocol for the Internet community, and requests discussion and suggestions for improvements. Please refer to the current edition of the "IAB Official Protocol

Standards" for the standardization state and status of this protocol. Distribution of this memo is unlimited.

Abstract

The Point-to-Point Protocol (PPP) [1] provides a standard method of encapsulating Network Layer protocol information over point-to-point links. PPP also defines an extensible Link Control Protocol, which allows negotiation of a Quality Protocol for continuous monitoring of the viability of the link.

This document defines a protocol for generating Link-Quality-Reports.

This RFC is a product of the Point-to-Point Protocol Working Group of the Internet Engineering Task Force (IETF). Comments on this memo should be submitted to the ietf-pppeucdavis.edu mailing list. 
Table of Contents

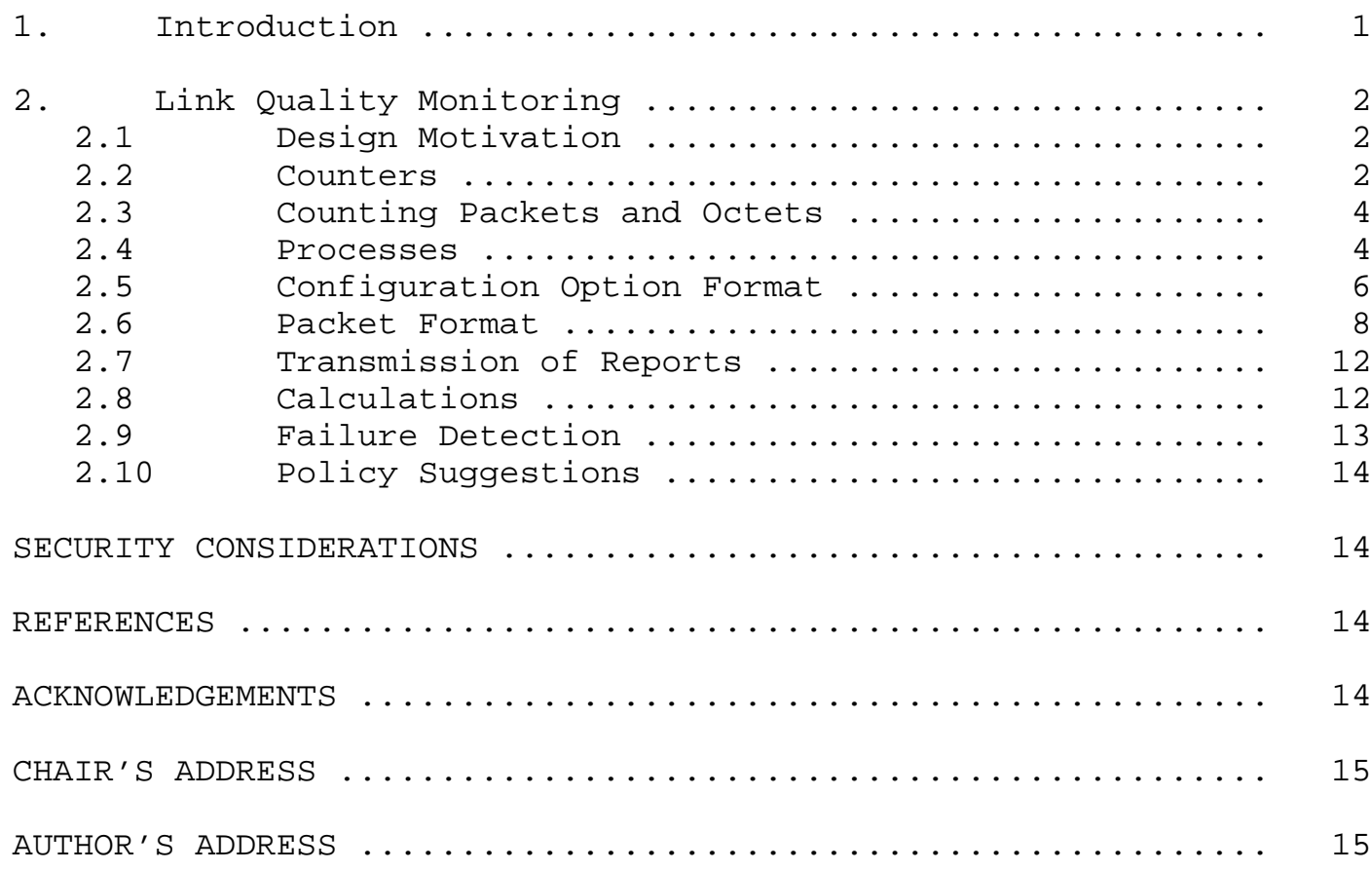


1. Introduction

PPP has three main components:

1. A method for encapsulating datagrams over serial links.

2. A Link Control Protocol (LCP) for establishing, configuring, and testing the data-link connection.

3. A family of Network Control Protocols (NCPs) for establishing and configuring different network-layer protocols.

In order to establish communications over a point-to-point link, each end of the PPP link must first send LCP packets to configure the data link during the Establishment phase. During the Authentication and Network-Layer Protocol phases, the link may be tested to determine if quality is sufficient for operation. This testing is completely optional.

If an implementation desires that the peer use some specific link quality monitoring protocol, then it MUST negotiate the use of that protocol using the Quality-Protocol Configuration Option during Link Establishment phase.

The negotiation mechanism is independent in each direction. However, if the peer agrees to send Quality-Protocol packets, it MUST correctly process such packets on reception, even if it does not request such packets or implement a monitoring policy. 


\section{Link Quality Monitoring}

Data communications links are rarely perfect. Packets can be dropped or corrupted for various reasons (line noise, equipment failure, buffer overruns, etc.). Sometimes, it is desirable to determine when, and how often, the link is dropping data. Routers, for example, may want to temporarily allow another route to take precedence. An implementation may also have the option of disconnecting and switching to an alternate link. The process of determining data loss is called "Link Quality Monitoring".

\subsection{Design Motivation}

There are many different ways to measure link quality, and even more ways to react to it. Rather than specifying a single scheme, Link Quality Monitoring is divided into a "mechanism" and a "policy". PPP fully specifies the "mechanism" for Link Quality Monitoring by defining the Link-Quality-Report (LQR) packet and specifying a procedure for its use. PPP does NOT specify a Link Quality Monitoring "policy" -- how to judge link quality or what to do when it is inadequate. That is left as an implementation decision, and can be different at each end of the link. Implementations are allowed, and even encouraged, to experiment with various link quality policies. The Link Quality Monitoring mechanism specification insures that two implementations with different policies may communicate and interoperate.

To allow flexible policies to be implemented, the PPP Link Quality Monitoring mechanism measures data loss in units of packets, octets, and Link-Quality-Reports. Each measurement is made separately for each half of the link, both inbound and outbound. All measurements are communicated to both ends of the link so that each end of the link can implement its own link quality policy for both its outbound and inbound links.

Finally, the Link Quality Monitoring protocol is designed to be implementable on many different kinds of systems. Although it may be common to implement PPP (and especially Link Quality Monitoring) as a single software process, multi-process implementations with hardware support are also envisioned. The PPP Link Quality Monitoring mechanism provides for this by careful definition of the LinkQuality-Report packet format, and by specifying reference points for all data transmission and reception measurements.

\subsection{Counters}

Each Link Quality Monitoring implementation maintains counts of the number of packets and octets transmitted and successfully received, 
and periodically transmits this information to its peer in a LinkQuality-Report packet.

These counters are similar to sequence numbers; they are constantly increasing to give a "relative" indication of the number of packets and octets communicated across the outbound link. By comparing the values in successive Link-Quality-Reports, an LQR receiver can compute the "delta" number of packets and octets successfully communicated across the link. Comparing these absolute numbers then gives an indication of a link's quality. Relative numbers, rather than absolute, are transmitted because they greatly simplify link synchronization.

The Link-Quality-Report uses the Interface counters defined by SNMP MIB-II [2]. These counters are not initialized to any particular value when the LCP enters the Establishment phase.

In addition, the Link-Quality-Report requires the implementation of the following three unsigned, monotonically increasing counters which conform to the type and size requirements for SNMP MIB Counters [3].

\section{Out LQRS}

OutLQRs is a 32-bit counter which increases by one for each tranmitted Link-Quality-Report packet. This counter MUST be set to zero when the LCP enters the Establishment phase, and MUST NOT be reset until the LCP leaves the Termination phase. This counter is incremented before it is inserted into the LQR packet.

\section{InLQRS}

InLQRS is a 32-bit counter which increases by one for each received Link-Quality-Report packet. This counter MUST be set to zero when the LCP enters the Establishment phase, and MUST NOT be reset until the LCP leaves the Termination phase. This counter is incremented before it is inserted (in an implementation dependent fashion) into the LQR packet.

\section{InGoodOctets}

InGoodOctets is a 32-bit counter which increases by the number of octets in each successfully received Data Link Layer packet. Unlike the MIB ifInoctets, octets for frames which are counted in ifInDiscards and ifInErrors MUST NOT be counted. This counter MAY be set to any initial value when the LCP enters the Establishment phase, but MUST NOT be reset until the LCP leaves the Termination phase. 


\subsection{Counting Packets and Octets}

The intent of the counters is to provide an indication of the amount of information passing over the link, rather than an actual measurement of the total bandwidth used. This specification is designed to yield the same count in various circumstances, such as when a separate device provides the framing and escaping mechanisms invisibly to the implementation, or a synchronous-to-asynchronous converter in the link changes between mechanisms.

All octets which are included in the FCS calculation MUST be counted, including the packet header, the information field, and any padding. The FCS octets MUST also be counted, and one flag octet per frame MUST be counted. All other octets (such as additional flag sequences, and escape bits or octets) MUST NOT be counted.

When inserting the packet and octet counts in the LQR, the counts MUST include the expected values for the LQR itself.

\subsection{Processes}

The PPP Link Quality Monitoring mechanism is described using a "logical process" model. As shown below, there are five logical processes duplicated at each end of the duplex link.

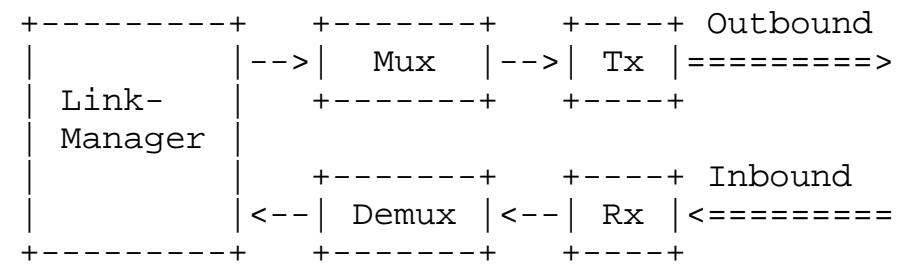

Link-Manager

The Link-Manager process transmits and receives Link-QualityReports, and implements the desired link quality policy. LQR packets are transmitted at a constant rate, which is negotiated by the LCP Quality-Protocol Configuration Option.

$\operatorname{Mux}$

The Mux process multiplexes packets from the various protocols (e.g., LCP, IP, XNS, etc.) into a single, sequential, and prioritized stream of packets. Link-Quality-Report packets MUST be given the highest possible priority to insure that link quality information is communicated in a timely manner. 
$\mathrm{Tx}$

The Tx process maintains the MIB counters ifoutUniPackets and ifoutoctets, and the internal counter OutLQRs, which are used to measure the amount of data which is transmitted on the outbound link. When Tx processes a Link-Quality-Report packet, it inserts the values of these counters into the corresponding PeerOut... fields of the packet. The Tx process MUST follow the Mux process so that packets are counted in the order transmitted to the link.

$\mathrm{Rx}$

The Rx process maintains the MIB counters ifInUnipackets, ifInDiscards, ifInErrors and IfInoctets, and the internal counters InLQRs and InGoodOctets, which are used to measure the amount of data which is received by the inbound link. When Rx processes a Link-Quality-Report packet, it inserts the values of these counters into the corresponding SaveIn... fields of the packet (in an implementation dependent manner).

Demux

The Demux process demultiplexes packets for the various protocols. The Demux process MUST follow the Rx process so that packets are counted in the order received from the link. 


\subsection{Configuration Option Format}

Description

Implementations MUST be prepared to receive the Quality-Protocol Configuration Option for the Link-Quality-Report. However, negotiation is not required. Negotiation is only necessary when the implementation wishes to ensure that the peer transmits LinkQuality-Reports as opposed to some other Quality-Protocol, or else to prevent the peer from maintaining its own timer, or else to establish a maximum time between transmissions of Link-QualityReports.

A summary of the Quality-Protocol Configuration Option format to negotiate the Link-Quality-Report is shown below. The fields are transmitted from left to right.

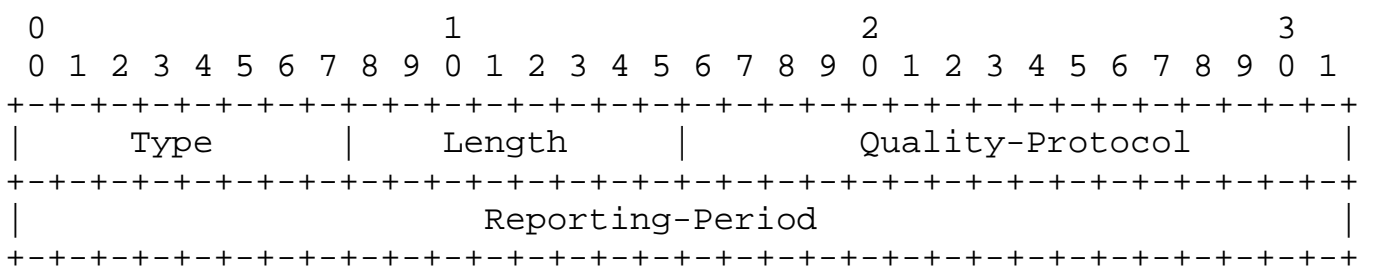

Type

4

Length

8

Quality-Protocol

c025 (hex) for Link-Quality-Report

Reporting-Period

The Reporting-Period field is four octets and indicates the maximum time in hundredths of seconds between transmission of packets. The peer MAY transmit packets at a faster rate than that which was negotiated.

A value of zero indicates that the peer does not need to maintain a timer. Instead, the peer generates a LQR immediately upon receiving a LQR. A value of zero MUST be Nak'd by the peer with 
an appropriate non-zero value when that peer has sent or will send a Configure-Request packet containing the Quality-Protocol Configuration Option for the Link-Quality-Report with a zero Reporting-Period. 


\subsection{Packet Format}

Exactly one Link-Quality-Report packet is encapsulated in the Information field of PPP Data Link Layer frames where the protocol field indicates type hex c025 (Link-Quality-Report). A summary of the LQR packet format is shown below. The names of the fields are relative to the packet receiver, since it is the receiver who requested the packet in the Configuration Option. The fields are transmitted from left to right.

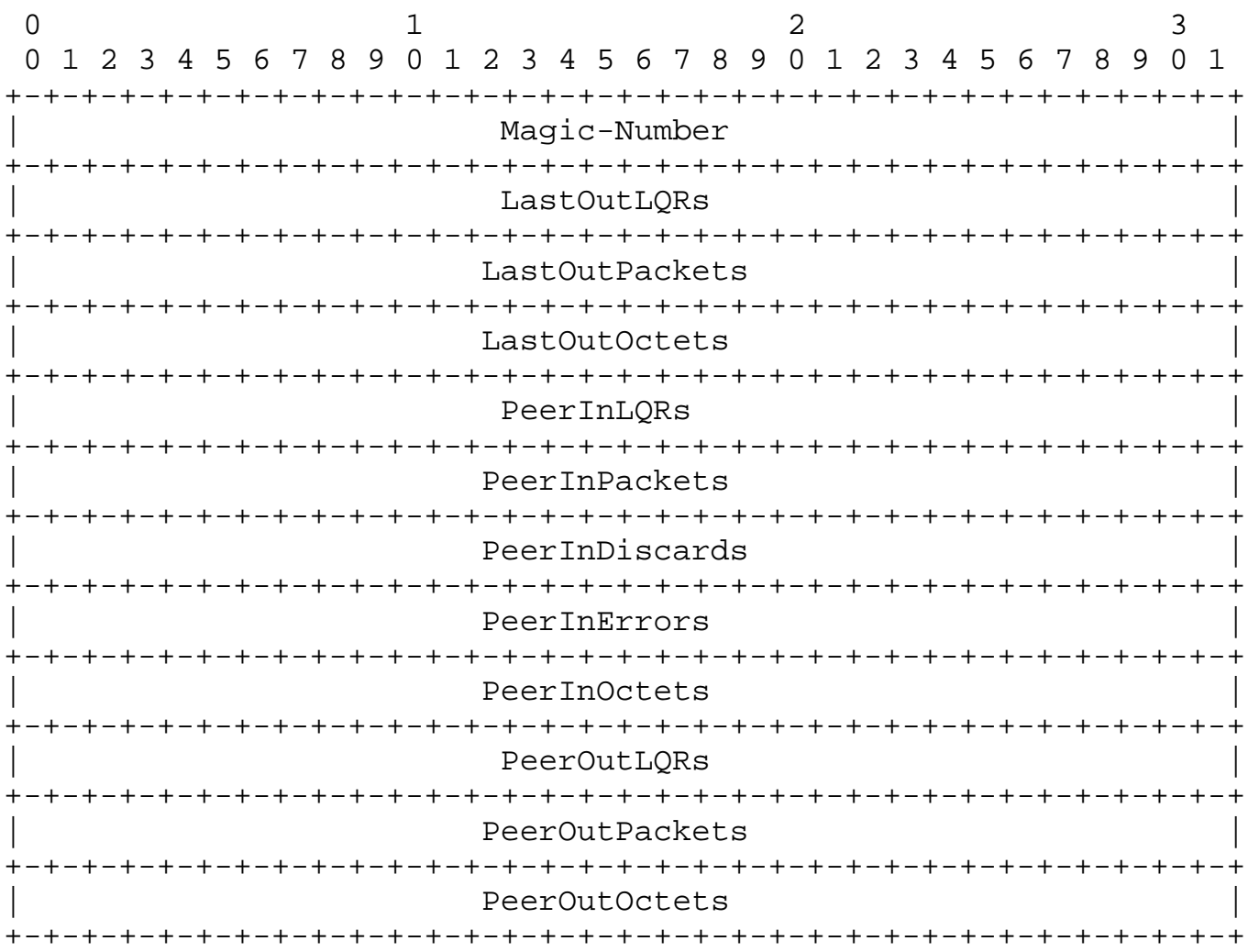

The following fields are not actually transmitted over the inbound link. Rather, they are logically appended (in an implementation dependent manner) to the packet by the implementation's Rx process.

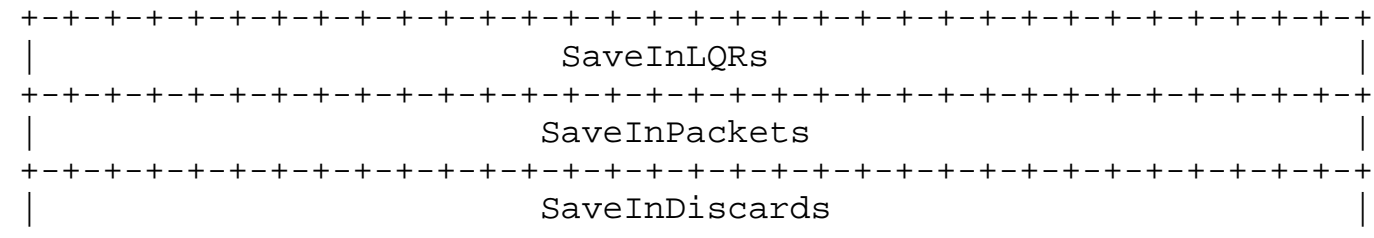




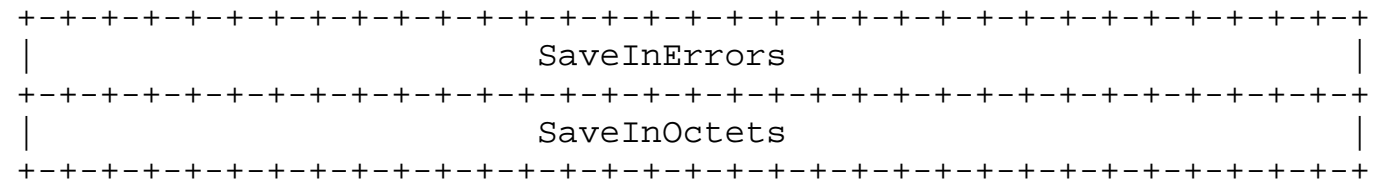

Magic-Number

The Magic-Number field is four octets and aids in detecting links which are in the looped-back condition. Unless modified by a Configuration Option, the Magic-Number MUST be transmitted as zero and MUST be ignored on reception. If Magic-Numbers have been negotiated, incoming LQR packets SHOULD be checked to ensure that the local end is not seeing its own Magic-Number and thus a looped-back link. See the Magic-Number Configuration Option for further explanation.

\section{LastoutLQRS}

The LastoutLQRs field is four octets, and is copied from the most recently received PeerOutLQRs on transmission.

LastOutPackets

The LastoutPackets field is four octets, and is copied from the most recently received PeerOutPackets on transmission.

Lastoutoctets

The Lastoutoctets field is four octets, and is copied from the most recently received PeerOutoctets on transmission.

PeerInLQRS

The PeerInLQRs field is four octets, and is copied from the most recently received SaveInLQRs on transmission.

Whenever the PeerInLQRs field is discovered to be zero, the Lastout... fields are indeterminate, and the PeerIn... fields contain the initial values for the peer.

\section{PeerInPackets}

The PeerInPackets field is four octets, and is copied from the most recently received saveInPackets on transmission. 
PeerInDiscards

The PeerInDiscards field is four octets, and is copied from the most recently received SaveInDiscards on transmission.

PeerInErrors

The PeerInErrors field is four octets, and is copied from the most recently received SaveInErrors on transmission.

PeerInoctets

The PeerInoctets field is four octets, and is copied from the most recently received SaveInoctets on transmission.

PeerOutLQRS

The PeerOutLQRs field is four octets, and is copied from OutLQRs on transmission. This number MUST include this LQR.

PeerOutPackets

The Peeroutpackets field is four octets, and is copied from the current MIB ifoutUniPackets and ifOutNUniPackets on transmission. This number MUST include this LQR.

PeerOutoctets

The Peeroutoctets field is four octets, and is copied from the current MIB ifOutoctets on transmission. This number MUST include this LQR.

SaveInLQRS

The SaveInLQRs field is four octets, and is copied from InLQRs on reception. This number MUST include this LQR.

SaveInPackets

The SaveInPackets field is four octets, and is copied from the current MIB ifInUniPackets and ifInNUniPackets on reception. This number MUST include this LQR.

SaveInDiscards

The SaveInDiscards field is four octets, and is copied from the current MIB ifInDiscards on reception. This number MUST include this LQR. 


\section{SaveInErrors}

The SaveInErrors field is four octets, and is copied from the current MIB ifInErrors on reception. This number MUST include this LQR.

SaveInoctets

The SaveInoctets field is four octets, and is copied from the current InGoodOctets on reception. This number MUST include this $\mathrm{LQR}$.

Note that InGoodOctets is not the same as the MIB ifInOctets counter, as InGoodoctets does not include octets for packets which are discards or errors. 


\subsection{Transmission of Reports}

When the PPP Link Control Protocol has reached the Opened state, the Link Quality Monitoring process MAY commence sending Link-QualityReports. If a Protocol-Reject is received specifying a LQR packet, the LQM process MUST cease sending LQRS.

Usually, the LQR is transmitted when the LQR timer for the link expires. If no LQR timer is used, a LQR is generated upon receipt of an incoming LQR. The negotiation process ensures that at least one side of the link is using a LQR timer.

In addition, a LQR is generated whenever two successive LQRs are received which have the same PeerInLQRs value. This may indicate that a LQR has been missed, or that the implementation is sending at a significantly slower rate than the peer, or that the peer has accelerated LQR generation to better quantify errors on the link.

Whenever a LQR is sent, the LQR timer MUST be restarted.

\subsection{Calculations}

Each time a Link-Quality-Report packet is received from the inbound link, the Link-Manager can compare the associated fields. The fields of the previous LQR can be subtracted from the current LQR values to obtain an absolute "delta", which allows comparision of the changes seen by each end of the link.

If the received PeerInLQRs field is zero, the Lastout... fields are indeterminate, and the PeerIn... fields contain the initial values for the peer. No calculations using these fields can be performed at this time.

Implementation Note:

The following counters wrap to zero when their maximum value is reached. Care must be taken to ensure that correct "delta" calculations are performed at that time.

The LastoutLQRs field may be directly compared with the PeerInLQRS field to determine how many outbound LQRs have been lost.

The LastoutLQRs field may be directly compared with the OutLQRs counter to determine how many outbound LQRs are still in the pipeline.

The change in PeerInPackets may be compared with the change in LastoutPackets to determine the number of lost packets over the 
outgoing link.

The change in PeerInoctets may be compared with the change in Lastoutoctets to determine the number of lost octets over the outgoing link.

The change in SaveInPackets may be compared with the change in PeerOutPackets to determine the number of lost packets over the incoming link.

The change in SaveInoctets may be compared with the change in Peeroutoctets to determine the number of lost octets over the incoming link.

The change in the PeerInDiscards and PeerInErrors fields may be used to determine whether packet loss is due to congestion in the peer rather than physical link failure.

\subsection{Failure Detection}

When the link is operating well in both directions of the link, the LQR is superfluous. The maximum time interval for transmitting LQRS SHOULD be chosen to minimally interfere with active traffic.

When there is a measurable loss of data in either direction, if the overall throughput is adequate, conditions are not severe enough to warrant dropping the link. Sending LQRs faster will gain nothing, except to measure peaks in the loss rate. The time interval MUST be chosen to be long enough to have a good smoothing effect on the data, while short enough to ensure fast enough response to complete failure.

When the link is good incoming, but very bad outgoing, incoming LQRS indicate a high loss on the outgoing side of the link. Sending LQRs faster won't help, because they are probably lost on the way to the peer.

When the link is good outgoing, but very bad incoming, incoming LRQs will be frequently lost. In this case, LQRs SHOULD be sent at a faster rate. This primarily relies on the peer to make an informed policy decision. The peer will also send LQRs in response (due to the duplicate PeerInLQRs field), and some of those LQRs may successfully arrive.

When a LQR does not arrive within the time expected, or the LQR received indicates that the links are truly bad, at least one additional LQR SHOULD be sent. An algorithmic decision requires at least 2 round trip intervals. The loss rate could be transient, due 
to a heavily loaded link, or a lost outgoing LQR.

\subsection{Policy Suggestions}

Link-Quality-Report packets provide a mechanism to determine the link quality, but it is up to each implementation to decide when the link is usable. It is recommended that this policy implement some amount of hysteresis so that the link does not bounce up and down. One policy is to use a $\mathrm{K}$ out of $\mathrm{N}$ algorithm. In such an algorithm, there must be $K$ successes out of the last $N$ periods for the link to be considered of good quality.

Procedures for recovery from poor quality links are unspecified and may vary from implementation to implementation. A suggested approach is to immediately close all other Network-Layer protocols (i.e., cause IPCP to transmit a Terminate-Request), but to continue transmitting Link-Quality-Reports. Once the link quality again reaches an acceptable level, Network-Layer protocols can be reconfigured.

Security Considerations

Security issues are not discussed in this memo.

References

[1] Simpson, W., "The Point-to-Point Protocol", RFC 1331, May 1992.

[2] McCloghrie, K., and M. Rose, "Management Information Base for Network Management of TCP/IP-based internets: MIB-II", RFC 1213, March 1991.

[3] Rose, M., and K. McCloghrie, "Structure and Identification of Management Information for TCP/IP-based Internets", RFC 1155, May 1990 .

Acknowledgments

Some of the text in this document is taken from RFC 1172, by Drew Perkins of Carnegie Mellon University, and by Russ Hobby of the University of California at Davis.

Special thanks to Craig Fox (Network Systems), and Karl Fox (Morning Star Technologies), for design suggestions based on implementation experience. 
Chair's Address

The working group can be contacted via the current chair:

Brian Lloyd

Lloyd \& Associates

3420 Sudbury Road

Cameron Park, California 95682

Phone: (916) 676-1147

EMail: brian@ray.lloyd.com

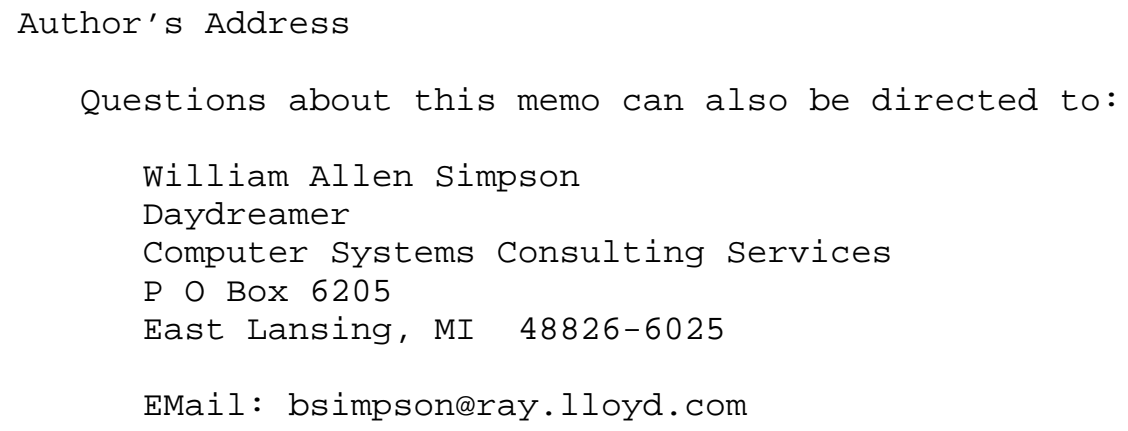

